\title{
Composition dependence of transition temperature in some ceramic superconductors
}

\author{
R K SINGH, DINESH VARSHNEY, N K GAUR and A C SHARMA* \\ School of Physics, Barkatullah University, Bhopal 462026, India \\ * School of Physics, Jiwaji University, Gwalior 474001 , India \\ MS received 15 June 1992; revised 4 November 1992

\begin{abstract}
The composition dependence of transition temperature in some ceramic superconductors $\left(\mathrm{La}_{2-x}(\mathrm{Ba}, \mathrm{Sr})_{x} \mathrm{CuO}_{4}\right)$ was studied by modifying our earlier approach and developing a Fourier-transformed effective potential which involves the effect of two-dimensional (2D) acoustic plasmons. This potential was used to obtain the pairing (electron-electron attraction) parameter $(\lambda)$, the averaged Coulomb repulsive parameter $\left(\mu^{*}\right)$ and the cut-off $2 \mathrm{D}$ acoustic plasmon frequency $\left(\omega_{c}\right)$ required to compute the superconducting transition temperature $\left(T_{c}\right)$ from the strong coupling theory. The variations of $T_{c}$ with compositions $(x)$ obtained for $\mathrm{La}_{2-x}(\mathrm{Ba}, \mathrm{Sr})_{x} \mathrm{CuO}_{4}$ show reasonably good agreement with experimental data.
\end{abstract}

Kevwords. Plasmons; phonons; transition temperature; coupling parameter; superconductors.

\section{Introduction}

The discovery of high temperature superconductivity (HTSC) in rare-earth (RE) cuprates (Bednorz and Muller 1986) has led to unprecedented research activity. The doping of transition and RE elements into the copper oxide ceramics plays a crucial role in determining their normal and superconducting properties. For instance, the phasetransition from normal to superconducting state, the stabilization of superconducting phase, and the maximum value of the critical temperature $\left(T_{c}\right)$ strongly depend on the doping concentration $(x)$ of these elements. Various superconducting properties of $\mathrm{La}_{2-x}(\mathrm{Ba} / \mathrm{Sr})_{x} \mathrm{CuO}_{4}$ and those of similar materials have earlier been investigated as a function of $x$ (Bednorz et al 1987; Dover et al 1987; Jha 1987; Shafer et al 1987; Weber 1987; Markert et al 1988; Moodenbaugh et al 1988; Torrance et al 1988; Seaman et al 1989; Tewari and Gumber 1990; Singh et al 1991).

Recently, Dover et al (1987) and Shafer et al (1987) measured $T_{c}$ as a function of $x$ in $\mathrm{La}_{2-x} \mathrm{Sr}_{x} \mathrm{CuO}_{4}$ system and found that $T_{c}$ first increased with $x$ and then decreased after reaching its maximum value at $x=0 \cdot 15$. Subsequently, Torrance et al (1988) observed that the variation of $T_{c}$ was below $5 \mathrm{~K}$ for $0.06 \leqslant x \geqslant 0.30$ in $\mathrm{La}_{2-x} \mathrm{Sr}_{x} \mathrm{CuO}_{4}$ with its maximum $\left(T_{c}=36 \mathrm{~K}\right)$ at $x=0.15$. However, it behaved like a normal metal at $x>0 \cdot 30$. Moodenbaugh et al (1988) also observed the variation of $T_{c}$ with $x$ in $\mathrm{La}_{2-x} \mathrm{Ba}_{x} \mathrm{CuO}_{4}$ system and found that $T_{c}$ had two maximum values, one at $x=0.09$ and another at $x=0 \cdot 15$. Further, Bednorz et al (1987) and Markert et al (1988) reported the variation of $T_{c}$ as a function of $x$ in $\mathrm{La}_{2-x} \mathrm{Ca}_{x} \mathrm{CuO}_{4}$ and $\mathrm{La}_{2-x} \mathrm{Na}_{x} \mathrm{CuO}_{4}$ superconductors, respectively. Motivated from these experimental observations, we thought it pertinent to perform an extensive theoretical analysis of the composition dependence of $T_{c}$ in $\mathrm{La}_{2-x}(\mathrm{Ba} / \mathrm{Sr})_{x} \mathrm{CuO}_{4}$.

Singh et al (1991) have recently computed the variation of $T_{c}$ with $x$ in $\mathrm{La}_{2-x}$ $(\mathrm{Ba} / \mathrm{Sr})_{x} \mathrm{CuO}_{4}$ superconductors using the weak coupling theory of Kresin (1987). 
Although their results are in good agreement with experimental data for the intermediate range of $x(0.06 \leqslant x \leqslant 0.31)$, the agreement is not so good for the lower and higher concentrations $(x)$. These deviations between experimental and earlier values of $T_{c}$ calculated by Singh et al (1991) might be due to the following limitations present in their interaction potential: (a) it does not take proper account of the involvement of electronic excitations (2D acoustic plasmons) in the pairing mechanism and (b) their formalism to calculate the cut-off frequency and the coupling parameter for plasmons does not properly include the effect of structural changes in $\mathrm{La}_{2-x}(\mathrm{Ba} / \mathrm{Sr})_{x} \mathrm{CuO}_{4}$ superconductors.

In this paper, we have attempted to eliminate the drawbacks of our earlier results (Singh et al 1991). The main features of our modified approach are (i) the transition temperature has been computed from the formalism based on the strong coupling theory of Eliashberg (1960) and (ii) the model parameters (coupling parameter $(\lambda)$, modified averaged Coulomb repulsion $\left(\mu^{*}\right)$, and upper cut-off $2 \mathrm{D}$ acoustic plasmon frequency $\left(\omega_{c}\right)$ ) are calculated by using the effective interaction potential (Jha 1987; Sharma 1989). The essentials of the potential formulation are presented in $\S 2$ and the computed results in $\S 3$.

\section{Theoretical formalism}

The change in concentration $(x)$ in $\mathrm{La}_{2-x}(\mathrm{Ba} / \mathrm{Sr})_{x} \mathrm{CuO}_{4}$ superconductors introduces two types of changes in the system. The first is in carrier states and free-carrier density $\left(n_{c}\right)$ and the other in crystal structure. The usual bulk properties such as phonon, plasmons, etc. are not expected to change significantly for small variations in $x$. Therefore, one cannot expect that BCS type theory, based on the pairing of charge carriers by the exchange of only phonons, is capable of explaining the drastic change in $T_{c}$ as a function of $x$. This, in turn, suggests the involvement of the collective electronic excitations (plasmons, Bozovic 1990) along with phonons in the pairing of the current carrying charge carriers.

The present paper thus includes the effects of electronic excitations (2D acoustic plasmons) in $V_{\text {eff }}$ used for the evaluation of $T_{c}$ and potential parameters $\left(\lambda, \mu^{*}\right.$ and $\left.\omega_{c}\right)$ in $\mathrm{La}_{2-x}(\mathrm{Ba} / \mathrm{Sr})_{x} \mathrm{CuO}_{4}$. The model interaction potential has been formulated for $\mathrm{La}_{2-x}(\mathrm{Ba} / \mathrm{Sr})_{x} \mathrm{CuO}_{4}$ and allied superconductors, based on the assumptions that (i) there is only one conducting $\mathrm{Cu}-\mathrm{O}$ plane per unit cell, (ii) the $\mathrm{Cu}-\mathrm{O}$ planes form an infinite array of planes along $c$-axis, (iii) the non-conducting planes between $\mathrm{Cu}-\mathrm{O}$ planes are considered as an uniform dielectric medium with a background dielectric constant $\left(\varepsilon_{\infty}\right)$, (iv) there are two types of acoustic plasmons corresponding to the electronic motion along and perpendicular to $a-b$ plane, (v) only long wave acoustic plasmons along $a-b$ motion of electrons (Bozovic 1990) take part in the pairing mechanism, and (vi) in the long wavelength limit, the Coulomb interactions between the charges on nearby $\mathrm{CuO}$ planes have a significant influence (Fetter 1974) on the interaction potential. In order to discuss the possible changes in the dielectric screening of these nearby planes, we have considered the screened Coulomb potential for a series of identical planes (see figure 1).

The Fourier-transformed effective interaction potential has been expressed as (Jha 1987; Sharma 1989)

$$
V_{\text {eff }}\left(q, q_{z}, \omega\right)=\frac{2 \pi e^{2} S\left(q, q_{z}\right)}{\varepsilon_{\infty} q \varepsilon\left(q, q_{z}, \omega\right)}
$$




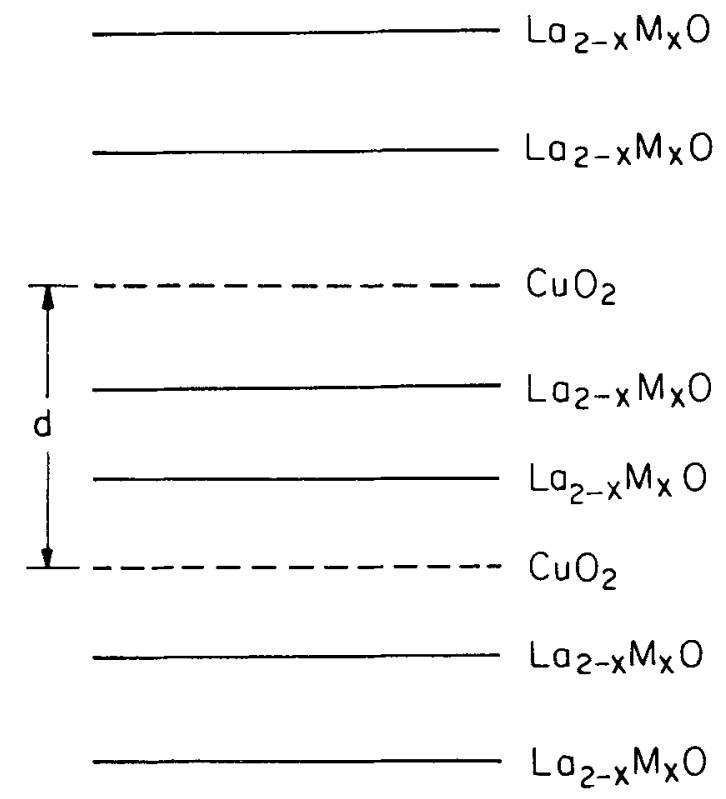

Figure 1. Schematic diagram of layered structure of cuprate superconductors.

in terms of the structure factor (Fetter 1974)

$$
S\left(q, q_{z}\right)=\frac{\sinh (q d)}{\cosh (q d)-\cos \left(q_{z} d\right)} \simeq \frac{q d}{\phi}
$$

for small values of the wave vector $(q)$ and $\phi=1-\cos \theta$ with $\theta$ as an angle between $q_{z}$ and $d$. Here, $d$ is the distance between the consecutive $\mathrm{Cu}-\mathrm{O}$ planes, $q$ and $q_{z}$, the components of the momentum along and perpendicular to the $\mathrm{Cu}-\mathrm{O}$ plane, and $\varepsilon_{\infty}$, the high frequency background dielectric constant of the surrounding material.

In the limit $d \rightarrow \infty, V_{\text {eff }}$ reduces to a single plane result and $d \rightarrow 0$ to an ordinary three-dimensional electron potential. Thus, we can rewrite (1) as

$$
V_{\text {eff }}\left(q, q_{z}, \omega\right)=\frac{2 \pi e^{2} d}{\varepsilon_{\infty}} R\left(q, q_{z}, \omega\right)
$$

with

and

$$
R\left(q, q_{z}, \omega\right)=\frac{1}{\phi \varepsilon\left(q, q_{z}, \omega\right)}
$$

$$
\varepsilon\left(q, q_{z}, \omega\right)=1+P(q, \omega) S\left(q, q_{z}\right)
$$

as expressed by Sharma (1989). Here, $P(q . \omega)$ is the polarization function due to the charge carriers $(c)$ and the ions $(i)$ expressed as

$$
\begin{aligned}
P(q, \omega) & =P_{c}(q, \omega)+P_{i}(q, \omega) \\
& =\frac{2 \pi e^{2} n_{c} q}{m^{*} \varepsilon_{\infty}\left[\left(q^{2} v_{F}^{2} / 2\right)-\omega^{2}\right]}-\frac{\Omega_{i}^{2} q d}{2 \varepsilon_{\infty} \omega^{2}},
\end{aligned}
$$

where $n_{c}$ and $v_{F}$, being the 2D carrier density and Fermi velocity of charge carriers, respectively. 
Using (3) to (7) we can obtain the value of the function $R\left(q, q_{2}, \omega\right)$ as

$$
R\left(q, q_{z}, \omega\right)=\frac{\omega^{2}}{\phi \omega(\omega+i \delta)-B q^{2}}
$$

with

$$
B=2 d\left[\frac{v_{F}^{2}}{2 a^{*}}+\frac{\Omega_{i}^{2} d}{4 \varepsilon_{\infty}}\right]
$$

where $\omega$ is the frequency, $\Omega_{i}$, the usual three-dimensional ionic plasma frequency and $a^{*}$, the effective Bohr radius.

The effective interaction between the charge carriers in a given conducting $\mathrm{Cu}-\mathrm{O}$ layer can now be expressed as

$$
V_{\text {eff }}(q, \omega)=\frac{d}{2 \pi} \int_{-\pi / d}^{+\pi / d} V_{\text {eff }}\left(q, q_{z}, \omega\right) \mathrm{d} q_{z}
$$

This integral can be evaluated by making use of (1) and (5) to get

$$
V_{\text {eff }}(q, \omega)=\frac{2 \pi e^{2} D(q, \omega) \sinh (q d)}{q \varepsilon_{\infty}|D(q, \omega)|\left\{\left|D^{2}(q, \omega)-1\right|\right\}^{1 / 2}}
$$

with

$$
D(q, \omega)=P(q, \omega) \sinh (q d)-\cosh (q d)
$$

The interaction potential, $V_{\text {eff }}(q, \omega)$, includes the effects of direct Coulomb repulsion as well as those of $2 \mathrm{D}$ acoustic plasmon and phonon modes. This interaction energy is attractive, if

$$
D(q, \omega)<0
$$

and is responsible for the pairing mechanism. For small $q$ values, the attractive interaction from (11) is given by

$$
V_{\text {eff }}(q, \omega)=\frac{\sqrt{2 \pi e^{2} d}}{\{|q d P(q, \omega) R(q, \omega)|\}^{1 / 2}}
$$

in view of (5), (11) and (12). Here,

$$
R(q, \omega)=1+P(q, \omega) \frac{1}{2} q d .
$$

In the limit $d \rightarrow \infty$, the effective interaction potential given by (11) reduces to $2 D$ effective potential. Using the condition for attractive interaction (13), we may express

with

$$
V_{\text {eff }}^{\prime}(q, \omega)=\frac{2 \pi e^{2}}{q \varepsilon_{\infty}} R^{\prime}(q, \omega)
$$

$$
R^{\prime}(q, \omega)=1+P(q, \omega)
$$

An attractive potential energy for $R^{\prime}(q, \omega)<0$ from (13) to (17) is obtained. In terms of frequencies of the collective excitations of charge carriers and those of ions obtained from (14), we get the condition for attractive interaction energy as

$$
1+\frac{2\left(k_{1}-1\right)}{\left(2 k_{1}-1\right)\left[1-k_{1}\left(\omega / \omega_{\mathrm{pl}}\right)^{2}\right]}-\frac{1}{\left(2 k_{1}-1\right)\left(\omega / \omega_{\mathrm{ph}}\right)^{2}}<0
$$


with

and

$$
\begin{aligned}
& K_{1}=1+\frac{m^{*} e^{2} d}{\hbar^{2} \varepsilon_{\infty}}, \\
& \omega_{\mathrm{pb}}=\Omega_{i} q d / \sqrt{2}
\end{aligned}
$$

$$
\omega_{\mathrm{pl}}=q v_{F}\left(\left(k_{1} \sqrt{2}\right)\right)
$$

with $\omega_{\mathrm{ph}}$ and $\omega_{\mathrm{pl}}$ as the 2D acoustic phonon and plasmon frequencies, respectively. The second and third terms in (18) represent the contributions from the charge carriers and ions, respectively.

If we consider only phonons, (18) reduces to the following condition

$$
0<\omega<\omega_{\mathrm{ph}} /\left(\sqrt{2} k_{1}-1\right)
$$

and if one includes only $2 \mathrm{D}$ acoustic plasmons, (18) becomes

$$
\left[\omega_{\mathrm{pl}} / \sqrt{ } k_{1}\right]<\omega<\left[\omega_{\mathrm{pl}}\left(\sqrt{4} k_{1}-3\right) / k_{1}\right] \text {. }
$$

Now, it is obvious that the frequency range of an attractive potential obtained from (22) is too low to account for the high transition temperatures. While the frequency range obtained from (23) is adequate to reveal the high transition temperature, this also indicates the inclusion of $2 \mathrm{D}$ acoustic plasmon in $\mathrm{La}_{2-x}(\mathrm{Ba} / \mathrm{Sr})_{x} \mathrm{CuO}_{4}$ superconductors.

From (19) and (21), the plasma frequency dispersion relation can be written as

$$
\omega_{\mathrm{pl}}^{2}=\frac{q^{2} v_{F}^{2}}{2}+\frac{2 \pi n_{c} e^{2} q}{m^{*} \varepsilon_{\infty}},
$$

which is the same as that obtained by Fetter (1974) for free plasmon oscillations in the plane. It is seen from this equation that $\omega_{\mathrm{pl}}$ approaches a nondispersive wave with propagation speed, $u=v_{F} / \sqrt{ } 2$, for short wavelengths and $\omega_{\mathrm{pl}}$ varies like $q^{1 / 2}$ at long wavelengths $(q \rightarrow 0)$. Thus, it is found from these descriptions that 2D acoustic plasmon effects included in our potential are responsible for the high temperature superconductivity in the layered structure cuprates.

In the strong coupling theory of Eliashberg (1960), the superconducting transition temperature requires knowledge of the modified Coulomb repulsive parameter $\left(\mu^{*}\right)$ and coupling parameter $(\lambda)$ between attractive electrons. To obtain these, one needs to evaluate the following real and imaginary parts of the effective potential given by (3) as

$$
\begin{aligned}
& \text { Real } R\left(q, q_{z}, \omega\right)=\frac{\omega^{2}}{\left(\phi \omega^{2}-B q^{2}\right)}, \\
& \text { Imag. } R\left(q, q_{z}, \omega\right)=\frac{\pi B q^{2}}{\phi} \delta\left(\phi \omega^{2}-B q^{2}\right) .
\end{aligned}
$$

Here, $\delta\left(\phi \omega^{2}-B q^{2}\right)$ is the well-known Dirac delta function. In view of the above description, the averaged Coulomb repulsive parameter $(\mu)$ can be expressed as

$$
\mu=N(0) \int_{0}^{2 q_{F}}\left[\operatorname{Real} V\left(q, q_{z}, \omega\right) / q_{F}^{2}\right] q \cdot \mathrm{d} q
$$




$$
=\frac{2 \pi e^{2} d}{\varepsilon_{\infty}} N(0) \ln \left(\frac{2+D}{D}\right)
$$

with $D=2 d / a^{*}$ and $N(0)$ as the density of states per unit cell.

Finally, the modified Coulomb repulsive parameter $\left(\mu^{*}\right)$ representing the Coulomb electron-electron interaction is expressed as

$$
\mu^{*}=\frac{\mu}{1+\mu \ln \left(E_{F} / \omega_{c}\right)}=\frac{\mu}{1+\mu \ln \left(\pi \hbar^{2} n_{c} / m^{*} \omega_{c}\right)}
$$

with $E_{F}\left(=\pi \hbar^{2} n_{c} / m^{*}\right)$ being the Fermi energy and $\omega_{c}$, the cut-off frequency of $2 \mathrm{D}$ acoustic plasmons.

Using the Eliashberg theory for strongly coupled superconductors, the expression for the electron-electron pairing parameter $(\lambda)$ has been derived from the relation (Eliashberg 1960)

$$
\lambda=2 \int_{0}^{\omega_{c}} \alpha^{2}(\omega) \frac{F(\omega) \mathrm{d} \omega}{\omega}
$$

with $F(\omega)$ as the Boson density of states and $\alpha^{2}(\omega)$ as the coupling strength between fermions and bosons. In view of the present potential given in (3), we have written the function $\alpha^{2}(\omega) F(\omega)$ as

$$
\alpha^{2}(\omega) F(\omega)=\frac{\pi^{2} e^{2} d N(0) \omega^{2}}{\varepsilon_{\infty} B K_{F}^{2}\left(1-\cos \theta_{c}\right)\left(1-\bar{\omega}_{c}\right)^{1 / 2}}
$$

to obtain the value of $\lambda$ from (29) and (30) we get

$$
\lambda=\frac{\pi\left[1-\left(\left(1+\bar{\omega}_{c}\right) / 2\right)\left(1-\bar{\omega}_{c}\right)^{1 / 2}\right]}{\left[3+\left(\Omega_{i}^{2} a^{*} m^{*} d / 12 \varepsilon_{\infty} E_{F}\right)\right]\left(1-\cos \theta_{c}\right)},
$$

where $q_{c}\left(=2 K_{F}=\left(8 \pi n_{c}\right)^{1 / 2}\right)$ is the cut-off wave vector and the critical angle $\left(\theta_{c}\right)$ between $q_{z}$ and $d$ is $16^{\circ}$. The Fermi energy $\left(E_{F}\right)$ and the average cut-off $2 \mathrm{D}$ acoustic plasmon frequency $\left(\bar{\omega}_{c}\right)$ are given by

$$
\begin{aligned}
& E_{F}=\hbar^{2} K_{F}^{2} / 2 m^{*}=n_{c}\left(\pi \hbar^{2} / m^{*}\right), \\
& \bar{\omega}_{c}=\omega_{c} / E_{F}=m^{*} \omega_{c} / \pi \hbar^{2} n_{c} .
\end{aligned}
$$

The poles of (25) yield the frequency of $2 \mathrm{D}$ acoustic plasmon modes. The frequency of these modes can be divided into two parts, i.e. $\omega_{p}^{\| l}$ and $\omega_{p}^{\perp}$ corresponding to the motion of electron along and perpendicular to $a-b$ plane. We have, however, assumed that $\omega_{p}^{\perp}$ does not take part in pairing mechanism but only screens the electron motion along $a-b$ plane. In order to obtain the cut-off $2 \mathrm{D}$ acoustic plasmon frequency $\left(\omega_{c}\right)$ for which the effective potential remains negative, we have averaged (3) over $q$ and $q_{z}$ and hence obtained poles of the averaged effective potential to get

$$
\begin{aligned}
\omega_{c} & =0.556 K_{F} B^{1 / 2}\left[1-\left(\hbar K_{F} / E_{F}\right) B^{1 / 2} / 2 \cdot 83\right], \\
& =1.4\left[\left(n_{c} B\right)^{1 / 2}-0.28 m^{*} B\right] .
\end{aligned}
$$

Using the above expressions for $\lambda, \mu^{*}$ and $\omega_{c}$, the expression for the superconducting transition temperature $\left(T_{c}\right)$ can be written as (Ruvalds 1987)

$$
T_{c}=0.7 \omega_{c} \exp \left[-(1+\lambda) /\left(\lambda-\mu^{*}\right)\right]
$$


following the strong coupling theory of Eliashberg (1960). We have computed $T_{c}$ for different concentrations $(x)$ using the values of the potential parameters $\mu^{*}, \lambda$ and $\omega_{c}$ obtained from (28), (31) and (34) from the knowledge of $n_{c}\left(=n_{0} \times 10^{14} \mathrm{~cm}^{-2}\right)$ determined as a function of composition $(x)$ as the values of $n_{0}$ are reported (Hasegawa et al 1987; Ong et al 1987; Que et al 1987) for Ba and Sr-doped superconductors for the range $0 \leqslant x \leqslant 0 \cdot 3$. The results on $T_{c}$ thus computed by us as anction of $x$ for $\mathrm{La}_{2-x}(\mathrm{Ba} / \mathrm{Sr})_{x} \mathrm{CuO}_{4}(0 \leqslant x \leqslant 0 \cdot 3)$ have been presented and discussed below.

\section{Results and conclusion}

In order to calculate $T_{c}$ as discussed above, we have used the values of the effective mass $m^{*}$ equal to $6 m_{e}$ and $5 m_{e}$ for $\mathrm{La}_{2-x} \mathrm{Ba}_{x} \mathrm{CuO}_{4}$ and $\mathrm{La}_{2-x} \mathrm{Sr}_{x} \mathrm{CuO}_{4}$, respectively. The values of high frequency background dielectric constant $\left(\varepsilon_{\infty}\right)$ appearing in (28), (31) and (34) have been obtained as 4.5 as reported by Bozovic (1990) and Mahan and $\mathrm{Wu}$ (1989) for $\mathrm{Ba}$ and $\mathrm{Sr}$ doped superconductors. The values of the model parameters, particularly $\lambda$ and $\omega_{c}$, obtained as discussed earlier, have been plotted against the composition $(x)$ in figures 2 and 3 for $\mathrm{La}_{2-x} \mathrm{Ba}_{x} \mathrm{CuO}_{4}$ and $\mathrm{La}_{2-x} \mathrm{Sr}_{x} \mathrm{CuO}_{4}$, respectively. It is seen from figure 2 that $\lambda$ remains almost constant, while $\omega_{c}$ first increases and decreases after reaching the first maximum once a minimum value of $x=0.10$ is attained. It again increases and reaches a second maximum in the range of $x$ from $0 \cdot 10$ to $0 \cdot 25$. We thus find two peaks when $\omega_{c}$ is plotted as a function of $x$ for $\mathrm{La}_{2-x} \mathrm{Ba}_{x} \mathrm{CuO}_{4}$. Figure 3 shows that the values of $\lambda$ are almost constant throughout the range of $x$, while the values of $\omega_{c}$ first increase up to $x=0.20$

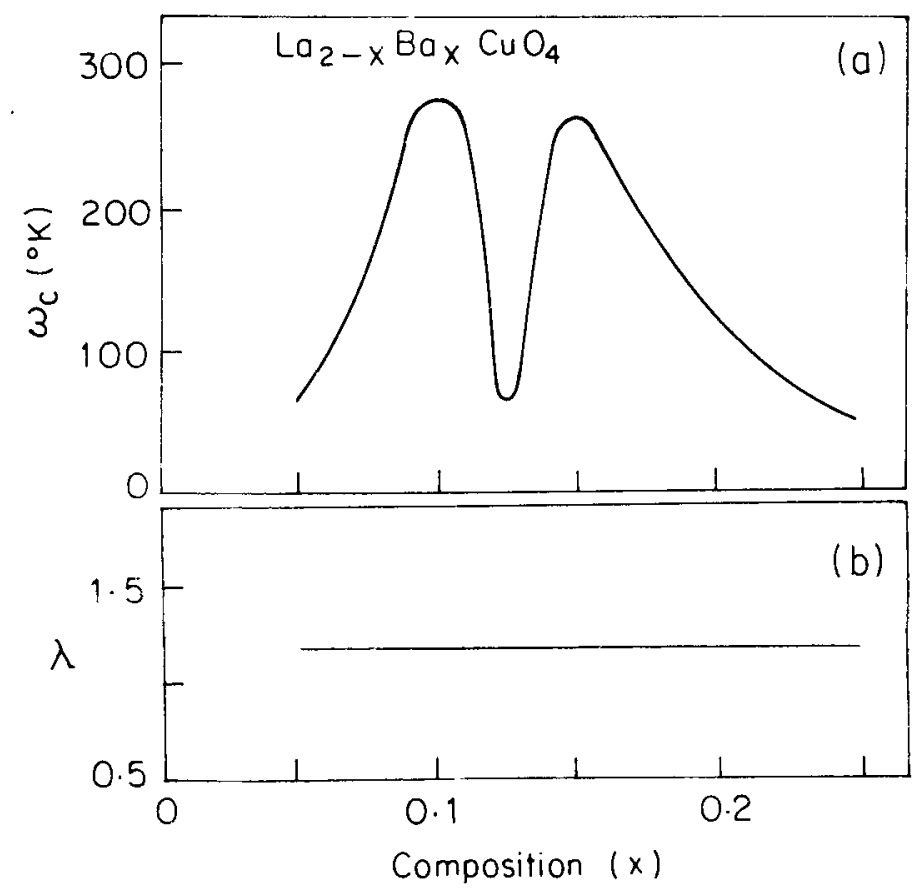

Figure 2. Variation of the parameters $\omega_{c}$ and $\lambda$ with composition $(x)$ shown in a and $\mathbf{b}$, respectively. 


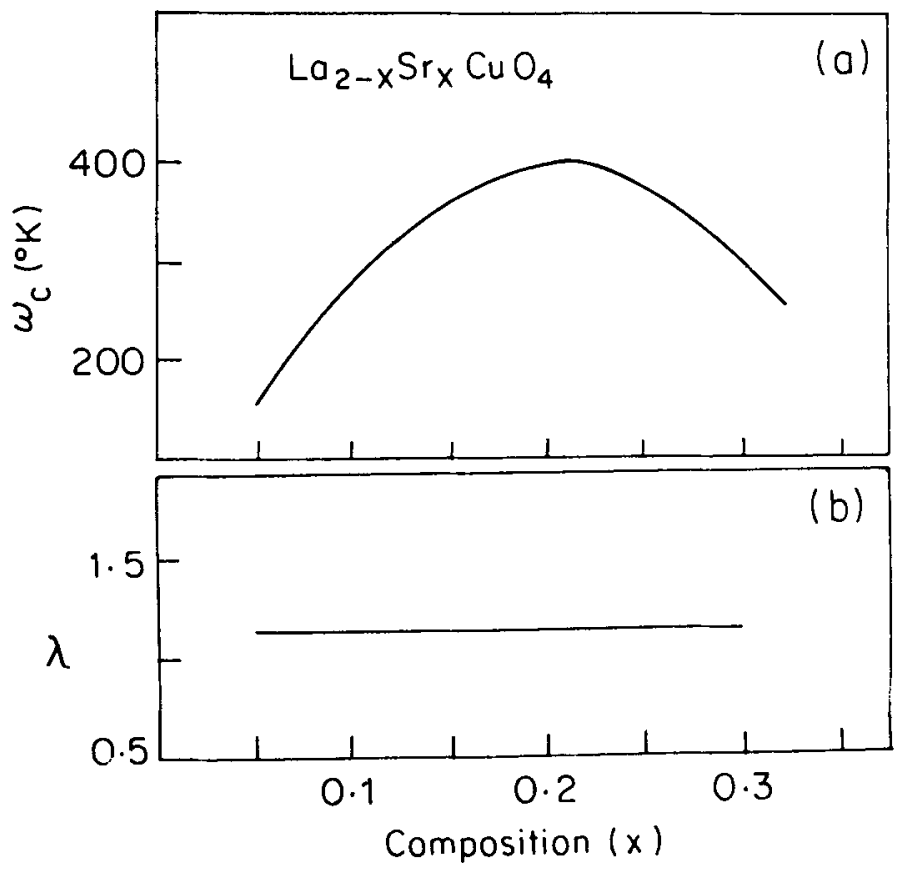

Figure 3. Variation of the parameters $\omega_{c}$ and $\lambda$ with composition $(x)$ shown in and $\mathbf{b}$, respectively.

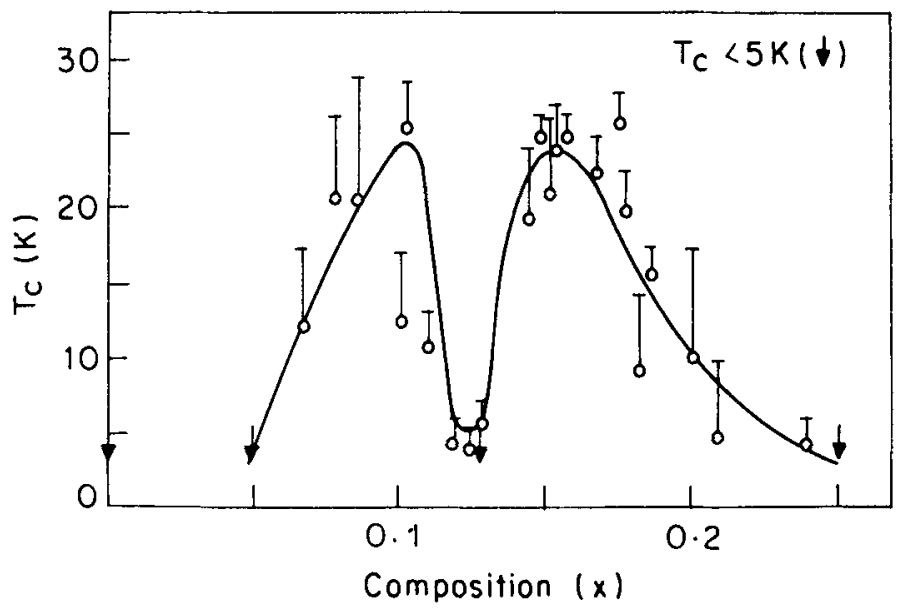

Figure 4. Variation of transition temperature $\left(T_{c}\right)$ with composition $(x)$. The circles $(J)$ are the experimental data taken from Moodenbaugh et al (1988).

and then gradually decrease with the increase of $x$. The characteristic of $\omega_{c}$ with $x$ for $\mathrm{La}_{2-x} \mathrm{Sr}_{x} \mathrm{CuO}_{4}$ is in keeping with the measured $\omega_{c}$ at different $x$ by Tajima et al (1988) and Suzuki (1989). However, such experimental data are not available for $\mathrm{La}_{2-x} \mathrm{Ba}_{x} \mathrm{CuO}_{4}$. The values of $\lambda \geqslant 1.0$ obtained by us at different concentrations $(x)$ indicate that the coupling due to $2 \mathrm{D}$ acoustic plasmons is strong in nature.

The values of $T_{c}$, computed by us using the model parameters $\lambda, \mu^{*}$ and $\omega_{c}$ obtained for different $x$ as discussed earlier, have been plotted as functions of $x$ in figures 4 


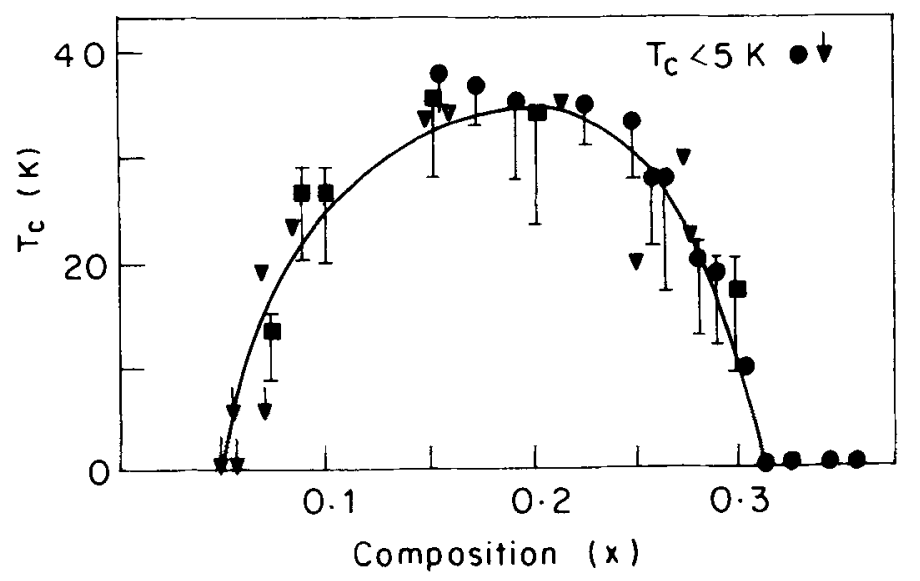

Figure 5. Variation of transition temperature $\left(T_{c}\right)$ with composition $(x)$. The $\boldsymbol{T}, \boldsymbol{\nabla}$ and are taken from Dover et al (1987). Shafer et al (1987) and Torrance et al (1988), respectively. The values of $T_{c}$ indicated by $\downarrow$ and $P$ are below $5 \mathrm{~K}$.

and 5 along with their experimental values for $\mathrm{La}_{2-x} \mathrm{Ba}_{x} \mathrm{CuO}_{4}$ (Moodenbaugh 1988) and $\mathrm{La}_{2-x} \mathrm{Sr}_{x} \mathrm{CuO}_{4}$ (Dover et al 1987; Shafer et al 1987; Torrance et al 1988), respectively. It is seen from these figures that our calculated values of $T_{c}$ are in a reasonably good agreement with their experimental values. Figure 4 shows that there are two maxima: one near composition $x=0.10$ with $T_{\varepsilon}=24 \mathrm{~K}$ and another near $x=0.16$ with $T_{c}=26 \mathrm{~K}$. These maxima of $T_{c}$ correspond to the recently observed peaks by Moodenbaugh et al (1988) at $x=0.10$ and 0.17 with $T_{c}=26$ and $26.5 \mathrm{~K}$, respectively. In $\mathrm{La}_{2-x} \mathrm{Ba}_{x} \mathrm{CuO}_{4}$ superconductor, we have obtained an anomalous dip around $x=0.12$ besides two maxima. This indicates that above and below the Ba composition $x=0 \cdot 12$, there are two superconducting phases and hence double transition reflects the intrinsic change of the electronic states around $x=0 \cdot 12$. It is thus almost clear that at $x=0 \cdot 12$, a structural transformation occurs from high temperature tetragonal to low temperature orthorhombic phase. The reduction of $T_{c}$ near $x=0 \cdot 12$, is related to a significant change in the electronic state. This seems to be an interesting feature in $\mathrm{La}_{2-x} \mathrm{Ba}_{x} \mathrm{CuO}_{4}$ superconductor and deserves detailed exploration.

We notice from figure 5 that there is a maximum for $T_{\varepsilon}$ in $\mathrm{La}_{2-x} \mathrm{Sr}_{x} \mathrm{CuO}_{4}$ near $x=0.15$ with $T_{c}=36 \mathrm{~K}$, which is in good agreement with the experimental results (Dover et al 1987; Shafer et al 1987; Torrance et al 1988). It is also noted from figure 5 that our calculated values of $T_{c}$ are closer to zero for $x \leqslant 0.06$ and $x \geqslant 0.30$. This feature is in keeping with the experimental data (Dover et al 1987; Shafer et al 1987; Torrance et al 1988). It can also be seen from this figure that $\mathrm{La}_{2-x} \mathrm{Sr}_{x} \mathrm{CuO}_{4}$ is superconducting only for $0.06 \leqslant x \leqslant 0.30$ according to our calculations, while Torrance et al (1988) have reported $T_{c}<5 \mathrm{~K}$ near $0.06 \leqslant x \geqslant 0.3$. We thus find that our calculations, based on the mechanism of pairing of charge carriers by the exchange of $2 \mathrm{D}$ acoustic plasmons, satisfactorily explain the observed variations of $T_{c}$ with composition $(x)$ in both $\mathrm{La}_{2-x}(\mathrm{Ba} / \mathrm{Sr})_{x} \mathrm{CuO}_{4}$ superconductors.

We may further add that the theoretical work of Weber (1987) on the calculation of $T_{c}$ for $\mathrm{La}_{2-x} \mathrm{Sr}_{x} \mathrm{CuO}_{4}$, which is based on the pairing of charge carriers by exchange of phonons only, does not exhibit the entire trend of the experimentally observed $T_{c}$ as a function of $x$. It is, therefore, obvious that the inclusion of the effects of electronic 
excitations (2D acoustic plasmons) in the calculation of $T_{c}$ is essential to describe the composition ( $x$ ) dependence of $T_{c}$ in $\mathrm{La}_{2-x}(\mathrm{Ba} / \mathrm{Sr})_{x} \mathrm{CuO}_{4}$ ceramic superconductors for entire range $(0 \leqslant x \leqslant 0 \cdot 3)$. Another important point which emerges from our calculations and those of Weber (1987) is that for $0.06 \leqslant x \leqslant 0.15$, the pairing by exchange of electronic excitations (2D acoustic plasmons) seems to dominate over the pairing by exchanged phonons. On the other hand, for $x>0.15, T_{c}$ has decreasing trend and hence the phonon mechanism seems to dominate over the electronic excitation (2D acoustic plasmons) mechanism in charge-carrier pairing.

In conclusion, we have succeeded in predicting the observed effect of $\mathrm{Ba}$ and $\mathrm{Sr}$ doping on the transition temperature $\left(T_{c}\right)$ in $\mathrm{La}_{2-x}(\mathrm{Ba}, \mathrm{Sr})_{x} \mathrm{CuO}_{4}$ superconductors. Our calculations of $T_{c}$ are based on a model effective interaction potential which includes properly the effects of $2 \mathrm{D}$ acoustic plasmons in pairing mechanism. The drawbacks present in our earlier work (Singh et al 1991) have almost been eliminated in this paper. Our results on composition dependence of $T_{c}$ demonstrate that the carrier collective excitations ( $2 \mathrm{D}$ acoustic plasmons) play an important role in the pairing of charge-carriers, especially for lower values of $x$ in cuprate superconductors. Such investigations in the cases of other members of this family are in progress and the results will be reported subsequently.

\section{Acknowledgements}

The authors are thankful to the University Grants Commission, New Delhi for financial support. They are also thankful to Profs R Srinivasan (IUC, Indore), S S Jha (TIFR, Bombay), and T V Ramakrishnan (IISc., Bangalore) for many useful discussions.

\section{References}

Bednorz J G and Muller K A 1986 Z. Phys. B64 189

Bednorz J G, Muller K A and Takashige M 1987 Science 73236

Bozovic I 1990 Phys. Rev. B42 1969

Dover R B van, Cava R J, Batlogg B and Rietman E A 1987 Phys. Rev. B35 5337

Eliashberg G M 1960 Sov. Phys. (JETP) 11696

Fetter A 1974 Ann. Phys. 881

Hasegawa T, Koshio K, Aoki M, Ooba N, Kitazawa K, Fueki K, Uchida S and Tanaka S 1987 Jpn. J. Appl. Phys. 26 L337

Jha S S 1987 Pramana-J. Phys. 29 L615

Kresin V Z 1987 Phys. Rev. B35 3116

Mahan G D and Wu J W 1989 Phys. Rev. B39 265

Markert J T, Seaman C L, Zhou H and Maple M B 1988 Solid State Commun. 66 387; Am. Phys. Soc. 33556

Que W M and Kirzcenow G 1987 Solid State Commun. 641053

Moodenbaugh A R, Xu Y, Suenaga M, Folkerts T J and Shelton R N 1988 Phys. Rev. B38 4596

Ong N P, Wang Z Z, Clayhold J, Tarascon J M, Greene L H and Mckinnon W R 1987 Phys. Rev. B35 8807

Ruvalds J 1987 Phys. Rev. B35 8869

Seaman C L et al 1989 Physica C159 391

Shafer M W, Penney T and Olson B L 1987 Phys. Rev. B36 4047

Sharma A C 1989 Solid State Commun. 701171

Singh R K, Sharma A C, Gaur N K and Varshney D 1991 Bull. Mater. Sci. 141023

Suzuki M 1989 Phys. Rev. B39 2312

Tajima S, Nakahashi T and Tanaka S 1988 Physica C156 90

Tewari S P and Gumber P K 1990 Physica C168 647; C171 147

Torrance J B, Tokura Y, Nazzal A I, Bezinge A, Huang T C and Parkin S S P 1988 Phys. Rev. Lett. 611127

Weber W 1987 Phys. Rev. Lett. 581371 